http://jmscr.igmpublication.org/home/ ISSN (e)-2347-176x ISSN (p) 2455-0450 crossref DOI: https://dx.doi.org/10.18535/jmscr/v7i10.27

\title{
A Study of the Efficacy of Microneedling with Topical Autologous Platelet Rich Plasma in the Treatment of Acne Scars in a Tertiary Care Centre
}

\section{Priyadharsini $\mathbf{R}^{1}$, Kaviarasan $\mathrm{PK}^{2}$, Prasad $\mathrm{PVS}^{3}$, Kannambal $\mathrm{K}^{4 *}$, Poorana $\mathrm{B}^{5}$ Abhirami $\mathbf{C}^{6}$}

${ }^{1}$ Post Graduate, Department of Dermatology Venereology and Leprosy, Rajah Muthiah Medical College \& Hospital, Annamalai University, Chidambaram, India - 608002.

${ }^{2}$ Head of the Department, Department of Dermatology Venereology and Leprosy, Rajah Muthiah Medical College \& Hospital, Annamalai University, Chidambaram, India - 608002.

${ }^{3}$ Professor, Department of Dermatology Venereology and Leprosy, Rajah Muthiah Medical College \& Hospital, Annamalai University, Chidambaram, India - 608002.

${ }^{4}$ Associate professor, Department of Dermatology Venereology and Leprosy, Rajah Muthiah Medical College \& Hospital, Annamalai University, Chidambaram, India - 608002

${ }^{5}$ Assistant professor, Department of Dermatology Venereology and Leprosy, Rajah Muthiah Medical College \& Hospital, Annamalai University, Chidambaram, India - 608002

${ }^{6}$ Lecturer, Department of Dermatology Venereology and Leprosy, Rajah Muthiah Medical College \& Hospital, Annamalai University, Chidambaram, India - 608002

\section{*Corresponding Author}

Kannambal K

\begin{abstract}
Background: Acne scars poses a significant psychosocial morbidity in this era of aesthetic concern. Acne scars can be classified into three types-atrophic, hypertrophic \& keloidal. Atrophic acne scars are the most common type. Many treatments are available for treating post acne scars. Platelet-rich plasma $(P R P)$ may be useful in atrophic scars by promoting collagen deposition. Microneedling also releases growth factors, which initiates a cascade of wound healing resulting in collagen deposition. Hence, microneedling and PRP should act synergistically to improve acne scars.

Objective: To assess the efficacy of microneedling with topical autologous platelet rich plasma in the treatment of acne scars.

Material and Methods: The study enrolled 20 cases of acne scars. Microneedling with topical PRP was applied over acne scars. They received 4sequential treatments with an interval of 4 weeks between each session. At first visit, scars were graded according to Goodman and Baron's qualitative acne scar grading system. After 4 sessions objective assessment of physician scores of improvement were determined by Goodman and Baron's qualitative \&quantitative acne scar grading system.

Results: Male female ratio was 0.43:1. After completion of 4sessions, qualitative assessment revealed reduction by 3 grades in 12 patients (60\%) and Quantitative assessment showed 8 patients (40\%) with very good reduction. Photographs were taken at each visit and compared.

Conclusion: Microneedling with topical PRP was safe, superior and efficacious in treating acne scars.

Keywords: Acne scars, Platelet Rich Plasma, microneedling, Goodman and Baron's qualitative and quantitative acne scar grading system.
\end{abstract}




\section{Introduction}

Acne vulgaris is one of the most common skin disease affecting more than $90 \%$ of adolescents and adults with scarring being commonest sequelae. ${ }^{1}$ Severe acne results not only permanent scarring but also significant physical and psychosocial morbidity, especially in adolescents. This ultimately results in diminished quality of life. ${ }^{2}$ Scarring usually occurs as a consequence of healing of active acne.

Acne scars can be categorized clinically into three types based on whether there is net loss or gain of collagen as atrophic, hypertrophic and keloidal. ${ }^{3}$ Jacob et al classified atrophic acne scars into ice pick scars, rolling scars and boxcar scars based on the parameters of width, depth and three dimensional architecture. ${ }^{4}$ The prevalence of ice pick scar is $60 \%-70 \%$, box car scar $20 \%-30 \%$ and rolling scar $15 \%-25 \%$.

In the modern era, the management of acne scars is meteoric ranging from subcision, dermabrasion, chemical peeling, tissue augmentation using fillers, ablative and non ablative laser techniques. Though these techniques resulted in varying degrees of success, they have their own consequences and resultant adverse effects. Therefore there is a true demand for newer novel therapeutic modalities that are more efficacious, safe with minimal downtime. In this regard, a recently developed minimally invasive novel technique is autologous platelet rich plasma (PRP) especially in the treatment of atrophic acne scars.

\section{Role of PRP \& Microneedling in acne scars}

Platelet rich plasma is blood plasma with concentrated platelets. The concentrated platelets contain large reservoir of growth factors, especially epidermal growth factor (EGF), platelet derived growth factor (PDGF), transforming growth factor $\beta$ (TGF- $\beta$ ), vascular endothelial growth factor (VEGF) which initiates cellular growth, morphogenesis and accelerates the natural healing process. ${ }^{5}$

Microneedling is performed with a derma roller which is a drum- shaped roller with 192 fine micro needles in eight rows, $0.5-1.5 \mathrm{~mm}$ in length and $0.1 \mathrm{~mm}$ in diameter. The microneedles are synthesized by reactive ion etching technique on silicon or medical grade stainless steel. The needles pierce the stratum corneum and create microconduits (holes) without damaging the epidermis. $^{6}$

Hence, combining microneedling with platelet rich plasma has led to substantial improvement of acne scars with good aesthetic results and skin rejuvenation. This study is aimed to assess the efficacy of microneedling with topical autologous platelet rich plasma in the improvement of facial acne scars.

\section{Materials \& Methods}

The study was conducted in the Department of Dermatology, Venereology and Leprosy, Rajah Muthiah Medical College from November 2017 to September 2019. Ethical clearance was sought from the institutional ethical committee. Patients in the age group of 18-40 years with atrophic acne scars were selected. Exclusion criteria were patients with active acne, history of keloidal scars, bleeding disorders, active skin infection like warts, herpes and bacterial infection.

A total of twenty patients with acne scars were selected on the basis of inclusion and exclusion criteria. A written informed consent was taken from all the patients before enrolling them in the study. A detailed history of the patients as per the prepared questionnaire was taken along with photographs of each patient. Acne scars were classified according to Goodman and Baron's qualitative acne scar grading system as shown in table 1 and the results were assessed using Goodman and Baron's qualitative and quantitative acne scar grading system as shown in table $2 \mathrm{a}$ and 2b. Data obtained were tabulated in Microsoft office excel 2007. Descriptive statistics like mean and percentage were used to infer results.

\section{Autologous platelet rich plasma preparation and method of administration}

Autologous platelet rich plasma was obtained by a two stage centrifuging process. $10 \mathrm{ml}$ of patient's whole blood sample was drawn from the median 
cubital vein and collected in four sterile tubes each containing $1 \mathrm{ml}$ anticoagulant (Sodium citrate). The tubes were centrifuged at 1500 revolutions per minute for 10 minutes in the centrifugal machine. At the end of first spin, three layers were formed. The bottom layer consists of RBCs, the middle layer consists of platelets and WBCs and the top platelet-poor plasma layer. Only the supernatant plasma containing platelets were transferred into another sterile tube without anticoagulant. This tube was subjected to a second spin at 3000revolutions per minute for 20 minutes to obtain a platelet concentrate. After the second spin, approximately $3 / 4$ the of the supernatant will be discarded and platelet rich pellet will be resuspended in remaining amount of plasma. $10 \%$ calcium chloride will be added as platelet activator (1:9) i.e., $1 \mathrm{ml}$ of calcium chloride in 9 $\mathrm{ml}$ of PRP. PRP will be thus obtained.

Facial skin was disinfected with alcohol swab, then a topical anaesthetic cream was applied, left for 30 minutes. The skin was stretched and microneedling was carried out in vertical, horizontal and both diagonal directions for about 4- 5 times and platelet rich plasma was smeared over the area. Ice packs was applied over the treated area.Patients were advised to apply broad spectrum sunscreen, emollients and to avoid sun exposure.

\section{Clinical evaluation}

At initial visit (week 0) facial acne scars were graded based on Goodman and Baron's qualitative acne scar grading system. Objective assessment of Physician scores of improvement were determined by Goodman and Baron's qualitative and quantitative acne scar grading system by a side by side comparison of pre-operative and postoperative photographs taken at every sessions and at the end of 1 month after the last session.

Table1: Qualitative grading and examples of acne scars by Goodman and Baron ${ }^{7}$

\begin{tabular}{|c|c|c|c|}
\hline Grade & Level of disease & Characteristics & Examples of scars \\
\hline 1 & Macular disease & $\begin{array}{l}\text { Erythematous, hyper or hypopigmented flat marks visible to } \\
\text { patient or observer irrespective of distance. }\end{array}$ & $\begin{array}{l}\text { Erythematous, hyper- or } \\
\text { hypopigmented flat marks }\end{array}$ \\
\hline 2 & Mild disease & $\begin{array}{l}\text { Mild atrophy or hypertrophy that may not be obvious at } \\
\text { social distances of } 50 \mathrm{~cm} \text { or greater and may be } \\
\text { coveredadequately by makeup or the normal shadow of } \\
\text { shaved beard hair in males or normal body hair if extrafacial. }\end{array}$ & $\begin{array}{l}\text { Mild rolling, small soft } \\
\text { papular }\end{array}$ \\
\hline 3 & Moderate disease & $\begin{array}{l}\text { Moderate atrophic or hypertrophic scarring that is obvious at } \\
\text { social distances of } 50 \mathrm{~cm} \text { or greater and is not covered easily } \\
\text { by makeup or the normal shadow of shaved beard hair but is } \\
\text { still able to be flattened by manual stretching of the skin }\end{array}$ & $\begin{array}{l}\text { More significant rolling, } \\
\text { shallow "boxscar", mild to } \\
\text { moderate hypertrophic or } \\
\text { papular scars. }\end{array}$ \\
\hline 4 & Severe disease & $\begin{array}{l}\text { Severe atrophic or hypertrophic scarring that is obvious at } \\
\text { social distances of } 50 \mathrm{~cm} \text { or greater and is not covered easily } \\
\text { by make-up or the normal shadow of shaved beard hair in } \\
\text { males or body hair (if extrafacial) and is not able to be } \\
\text { flattened by manual stretching of the skin }\end{array}$ & $\begin{array}{l}\text { Punched out atrophic } \\
\text { (deep box scar), ice pick, } \\
\text { bridges and tunnels, gross } \\
\text { atrophy, dystrophic scars } \\
\text { significant hypertrophy or } \\
\text { keloid. }\end{array}$ \\
\hline
\end{tabular}

Table 2a: Quantitative grading of acne scars by Goodman and Baron ${ }^{8}$

\begin{tabular}{|l|c|c|c|}
\hline Grade or type & $\begin{array}{c}\text { Number of lesions } \\
\mathbf{1}(\mathbf{1 - 1 0})\end{array}$ & $\begin{array}{c}\text { Number of lesions 2 } \\
(\mathbf{1 1 - 2 0})\end{array}$ & $\begin{array}{c}\text { Number of lesions 3 } \\
(>\mathbf{2 0})\end{array}$ \\
\hline Milder scarring (1 point each) & 1 point & 2 points & 3 points \\
\hline Macular erythematous pigmented & & & \\
\hline Mildly atrophic dish-like & & & \\
\hline Moderate scarring (2 points each & 2 points & & \\
\hline Moderately atrophic, dish like & & & \\
\hline $\begin{array}{l}\text { Punched out with shallow bases small } \\
\text { scars (<5mm) }\end{array}$ & & & \\
\hline Shallow but broad atrophic areas & & & \\
\hline
\end{tabular}




\begin{tabular}{|c|c|c|c|}
\hline Severe scarring ( 3 points each & 3 points & 6 points & 9 points \\
\hline \multicolumn{4}{|c|}{$\begin{array}{l}\text { Punched out with deep but normal bases,small } \\
\text { scars }(<5 \mathrm{~mm})\end{array}$} \\
\hline \multicolumn{4}{|c|}{$\begin{array}{l}\text { Punched out with deep but abnormal } \\
\text { bases, small scars }(<5 \mathrm{~mm})\end{array}$} \\
\hline \multicolumn{4}{|c|}{ Linear or troughed dermal scarring } \\
\hline \multicolumn{4}{|l|}{ Deep, broad atrophic areas } \\
\hline Hyperplastic & 2 points & 4 points & 6 points \\
\hline Papularscars & Area $<5 \mathrm{~mm}^{2}$ & Area $5-20 \mathrm{~mm}^{2}$ & Area $>20 \mathrm{~cm}^{2}$ \\
\hline Keloidal/Hypertrophic scars & 6 points & 12 points & 18 points \\
\hline
\end{tabular}

Table 2b: Assessment of improvement using Goodman and Baron's quantitative acne scar grading system

\begin{tabular}{|l|l|}
\hline Grades & Improvement status \\
\hline $0-5$ & Minimal reduction in GSGS scores \\
\hline $5-10$ & Moderate reduction in GSGS scores \\
\hline $10-15$ & Good reduction in GSGS scores \\
\hline$>15$ & Very good reduction in GSGS scores \\
\hline
\end{tabular}

Figure 1: Microneedling with PRP

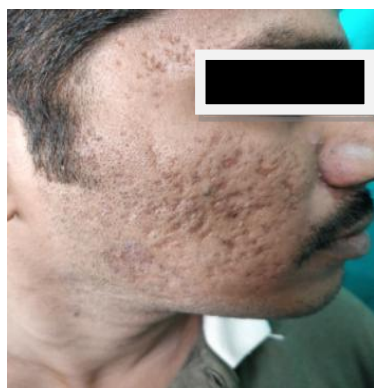

Pre treatment

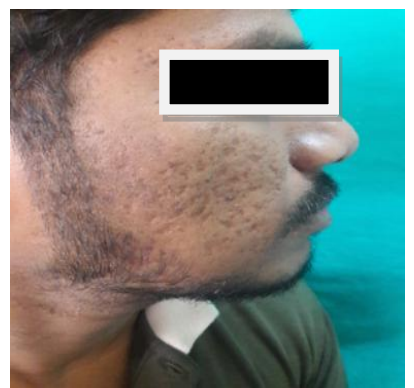

Post treatment

Figure 2: Microneedling with PRP

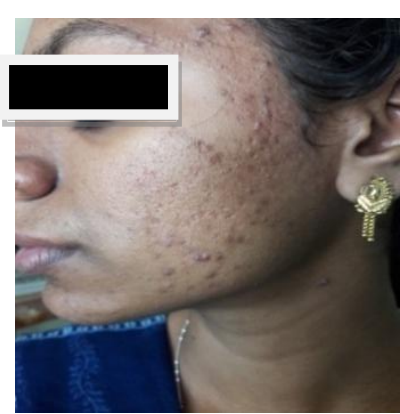

Pre treatment

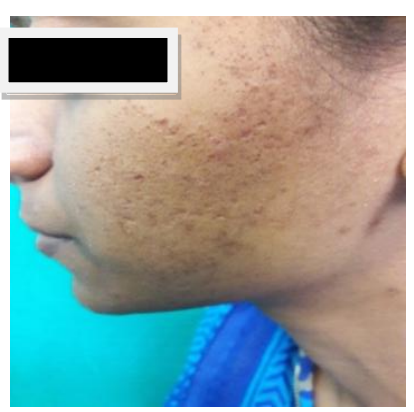

Post treatment

\section{Results}

The mean age of the patients was 25.4 years with the minimum age being 18 years and the maximum age being 35 years. Male: female ratio was 0.43:1. Acne scar duration varied from less than 1 year to more than 10 years. Majority of the patients $60 \%$ $(\mathrm{n}=12)$ gave history of scar duration of 1- 5 years, followed by $25 \%(n=5)$ with acne scar duration of 6-10 years, $10 \%(n=2)$ with duration of less than or equal to 1 year and $5 \%(n=1)$ with acne scar duration of more than 10 years. According to

Goodman and Baron acne scar grading scale, 10 patients presented with grade III (moderate) and 10 patients with grade IV (severe) on both sides of the face.

At the end of one month after last session (4 sessions), qualitative assessment showed out of 10patients with grade 3 acne scars, 7 patients (35\%) showed reduction by 2 grades and 3 patients $(15 \%)$ by 1 grade. Out of 10 patients with grade 4 acne scars, 5 patients (25\%) showed reduction by 3 grades, 3 patients $(15 \%)$ by 2 
grades and 2 patients (10\%) by 1 grade as shown in figure 3. Quantitative assessment showed 8 patients $(40 \%)$ with very good improvement, 6patients (30\%) with good improvement, 4 patients (20\%) with moderate and 2 patients $(10 \%)$ with minimal improvement as shown in figure 4.

Figure 3: Qualitative assessment of acne scars

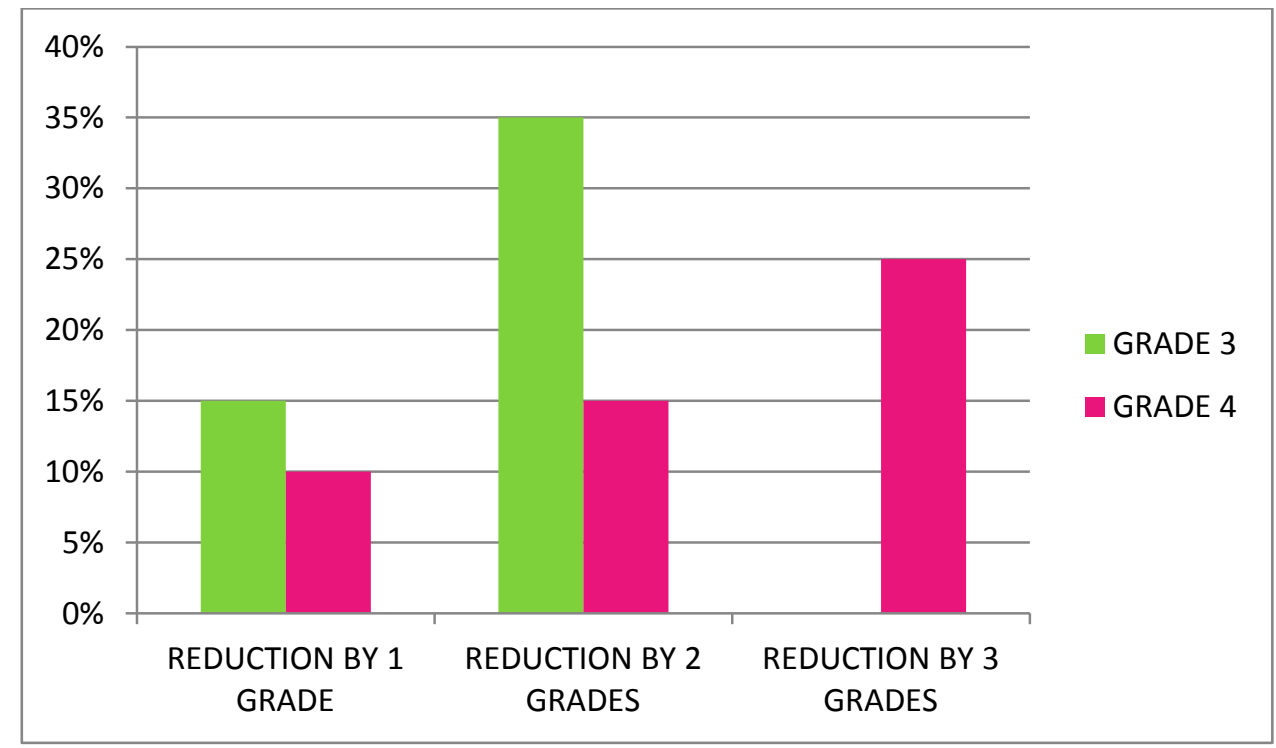

Figure 4: Quantitative assessment of acne scars

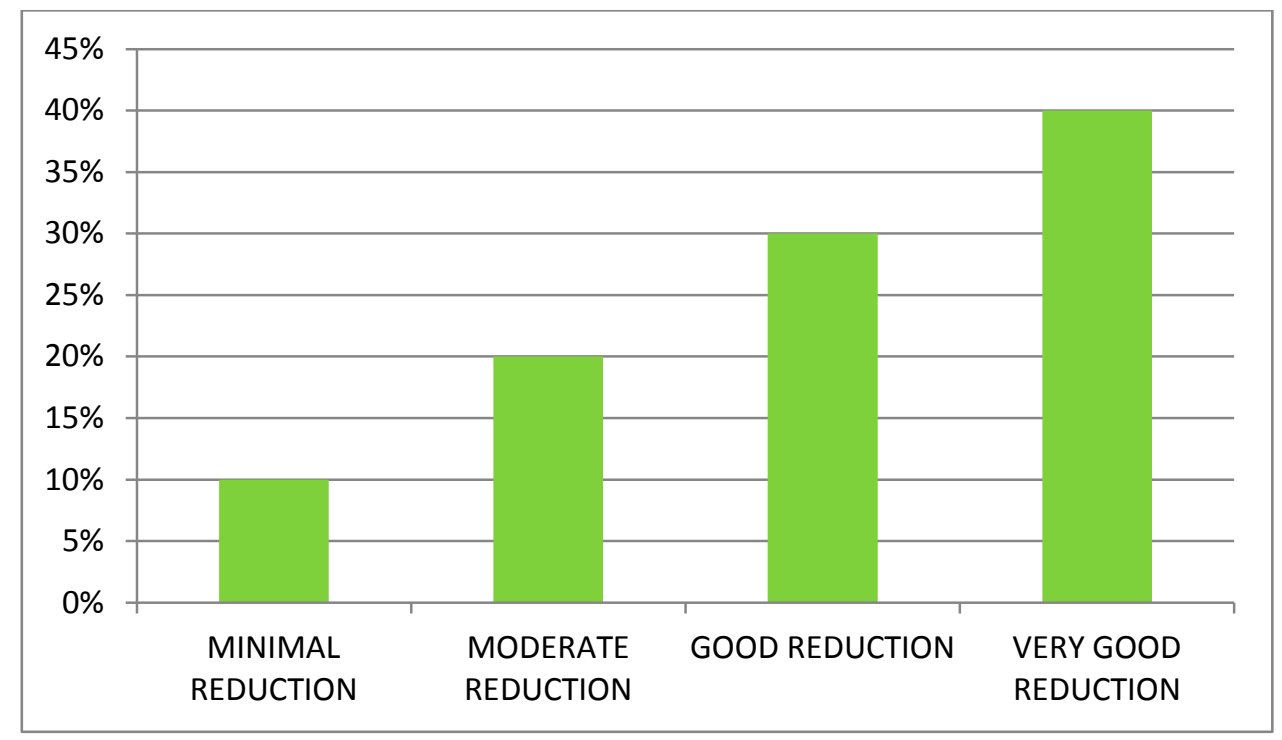

\section{Discussion}

Acne causes not only facial scarring but also it leads to significant psychosocial implications and emotional distress more so in adolescents. Various modalities have been used to treat scars, but limited efficacy and problematic side effects have restricted their application. PRP effectiveness in wound healing has prompted its use in the treatment of depressed facial scars, along with other available treatment modalities.
The possible mechanism of PRP in the reconstruction of a depressed scar is the induction of growth factors important in tissue remodeling, which promote connective tissue healing by up regulating collagen and protein synthesis. ${ }^{9}$ Growth factors present in PRP promotes \& accelerates tissue remodelling with the increased synthesis of collagen. ${ }^{10}$ So, PRP holds a promising role in soft tissue augmentation. 
Skin needling also called collagen induction therapy ${ }^{11}$ or needle dermabrasion is the technique of rolling a device comprising a barrel studded with hundreds of needles, which creates thousands of micropunctures in the skin to the level of papillary to mid-dermis. With this technique, the microneedling is usually continued until pinpoint bleeding point occurs, which initiates the complex cascade of growth factors that finally results in collagen production. ${ }^{12}$ Also it provides a clear conduit for agents to be absorbed more effectively through the upper layer of skin.

In our study, a statistically significant improvement was noted by combining PRP with microneedling. This finding could be attributed to the synergistic effects of both modalities on all types of atrophic acne scars. None of our patients showed any permanent pigmentary or textural changes. The only side effects noticed were redness and swelling of treated areas after each session of treatment, which subsided in 2 to 3 days.

\section{Limitation of the study}

The limitations of our study are small sample size, time constraint and long term follow - up of patients.

\section{Conclusion}

In conclusion, our study offers different, effective and safe therapeutic option for the treatment of atrophic acne scars. Combining microneedling with topically smeared platelet rich plasma is more effective in improving the quality of acne scars significantly. There were no significant side effects observed in this study. Three or four sessions of combination treatments are required for patients with moderate to severe acne scars. This technique is less expensive and is most likely useful in areas where there is limited access to laser technology. Further continuation of treatment might help to achieve a better outcome as scars only modify but never disappear completely.

\section{References}

1. Ranjan S, Gupta R, Gupta S. A Study of the Efficacy of Skin Needling and Platelet Rich Plasma in the Treatment of Acne Scars. Nepal J Dermatol Venereol Leprol 2017; 15:17-23.

2. Shah S, Mehta B, Borkar M, Aswani R. Study of safety and efficacy of autologous platelet rich plasma combined with fractional $\mathrm{CO} 2$ laser in the treatment of post acne scars: a comparative simultaneous split-face study. Int $J$ Res Med Sci2017;5(4):1344-1351.

3. Kravvas G, Al-Niaimi F. A systematic review of treatments for acne scarring. Part 1: Non-energy-based techniques. Scars Burn Heal. 2017;3:1-17.

4. Jacob CI, Dover JS, Kaminer MS. Acne scarring: A Classification system and review of treatment options. J Am Acad Dermatol 2001;45(1):109-117.

5. Dohan Ehrenfest DM, Rasmusson L, Albrektsson T. Trends Biotechnol. 2009 Mar;27(3):158-67.

6. Doddaballapur S. Microneedling with dermaroller. J Cutan Aesthet Surg 2009; 2(2):110-111.

7. Goodman GJ, Baron JA. Post acne scarring: A qualitative global scarring grading system. Dermatol Surg 2006; 32:1458-66.

8. Goodman GJ, Baron JA. Post acne scarring: A quantitative global scarring grading system. J Cosmet Dermatol 2006;5:48-52.

9. Ganio C, Tenewitz FE, Wilson RC, Moyles BG. The treatment of chronic nonhealing wounds using autologous platelet derived growth factors. J Foot Anke Surg 1993;32:263-268.

10. Lee JW, Kim BJ, Kim MN, Mun SK. The efficacy of autologous platelet rich plasma combined with ablative carbon dioxide fractional resurfacing for acne scars: a 
simultaneous split-face trial. Dermatol Surg 2011; 37(7): 931-8.

11. Fife D. Pratical evaluation and management of atrophic acne scars : tips for the general dermatologist. J Clin Aesthet Dermatol. 2011; 4: 50-57.

12. Fabbrocini G, Fardella N, Monfrecola A, et al. Acne scarring treatment using skin needling. Clin Exp Dermatol. 2009; 34: 874-879. 